\title{
Optimal mean first-passage time for a Brownian searcher subjected to resetting: Experimental and theoretical results
}

\author{
Benjamin Besga, ${ }^{1}$ Alfred Bovon $\odot,{ }^{1}$ Artyom Petrosyan $\odot,{ }^{1}$ Satya N. Majumdar, ${ }^{2}$ and Sergio Ciliberto ${ }^{1, *}$ \\ ${ }^{1}$ Université Lyon, Ens de Lyon, Université Claude Bernard, CNRS, Laboratoire de Physique, UMR 5672, F-69342 Lyon, France \\ ${ }^{2}$ LPTMS, CNRS, Université Paris-Sud, Université Paris-Saclay, UMR 8626, F-91405 Orsay, France
}

(Received 21 April 2020; accepted 17 June 2020; published 30 July 2020)

\begin{abstract}
We study experimentally and theoretically the optimal mean time needed by a free diffusing Brownian particle to reach a target at a distance $L$ from an initial position in the presence of resetting. Both the initial position and the resetting position are Gaussian distributed with width $\sigma$. We derived and tested two resetting protocols, one with a periodic and one with random (Poissonian) resetting times. We computed and measured the full firstpassage probability distribution that displays spectacular spikes immediately after each resetting time for close targets. We study the optimal mean first-passage time as a function of the resetting period and rate for different values of the ratio $b=L / \sigma$ and find an interesting phase transition at a critical value $b=b_{c}$. For $b_{c}<b<\infty$, there is a metastable optimum time which disappears for $b<b_{c}$. The intrinsic difficulties in implementing these protocols in experiments are also discussed.
\end{abstract}

DOI: 10.1103/PhysRevResearch.2.032029

When searching for a lost object in vain for a while, intuition tells us that maybe one should stop the current search and restart the search process all over again. The rationale behind this intuition is that a restart may help one to explore new pathways, thus facilitating the search process. This intuition has been used empirically before in various stochastic search algorithms (such as simulated annealing) to speed up the search process [1-4]. More recently, in the physics literature, this fact was demonstrated explicitly by studying the mean first-passage time (MFPT) for a single particle (searcher) to a fixed target in various models, in the presence of resetting (for a recent review, see Ref. [5]).

Searching a target via resetting is an example of the so-called intermittent search strategy [6] that consists of a mixture of short-range moves (where the actual search takes place) with intermittent long-range moves where the searcher relocates to a new place and starts a local search in the new place. More precisely, let there be a fixed target at some point in space and a particle (searcher) starts its dynamics from a fixed initial position in space. The dynamics of the particle may be arbitrary, e.g., it may be simply diffusive $[7,8]$. Resetting interrupts the natural dynamics of the particle either randomly at a constant rate $r[7,8]$ or periodically with a period $T[9,10]$, and sends the particle to its initial position. The dynamics starts afresh from the initial position after each resetting event. The MFPT to find a target, located at a fixed distance away from the initial position, typically

\footnotetext{
*sergio.ciliberto@ens-lyon.fr

Published by the American Physical Society under the terms of the Creative Commons Attribution 4.0 International license. Further distribution of this work must maintain attribution to the author(s) and the published article's title, journal citation, and DOI.
}

shows a unique minimum as a function of $r$ (or $T$ for periodical resetting). These studies have led to the paradigm that resetting typically makes the search process more efficient, and, moreover, there often exists an optimal resetting rate $r^{*}$ (or period $T^{*}$ ) that makes the search time minimal [7,8].

While this "optimal resetting" paradigm has been tested and verified in a large number of recent theoretical and numerical studies [9,11-28] (see also the review [5]), it still needs to be verified experimentally (see, however, the recent preprint [29], where the authors use a holographic optical tweezer setup. Their resetting protocols though are quite different from ours). The purpose of this Rapid Communication is to report an experimental realization using optical laser traps and a subsequent test and verification of this optimal resetting paradigm. The goal of the experiment is not just to mimic the theoretical models, but we will see that designing an experiment with a realistic resetting protocol is challenging. Moreover, our experimental protocol led us, in turn, to study different models that exhibit interesting and rich phenomena associated with resetting, namely metastability and a phase transition in the MFPT as a function of resetting rate and period.

Experimental setup. We have implemented an optimal search protocol with a Brownian particle which is periodically or randomly reset using optical tweezers [30]. We use a silica microsphere of radius $R=1 \mu \mathrm{m}( \pm 5 \%)$ immersed in pure water. The fluid chamber is designed to have very few particles in the measuring volume which allows us to take long measurements. A near-infrared laser with wavelength $\lambda=1064 \mathrm{~nm}$ is focused into the chamber through an oil immersion objective [Leica $63 \times$ and $1.4 \mathrm{nu}$ merical aperture (NA)] to create an optical trap. The particle is trapped in the $(x, y)$ plane by a harmonic potential with stiffness $\kappa$. The stiffness is controlled by changing the optical power in the chamber directly by laser current 

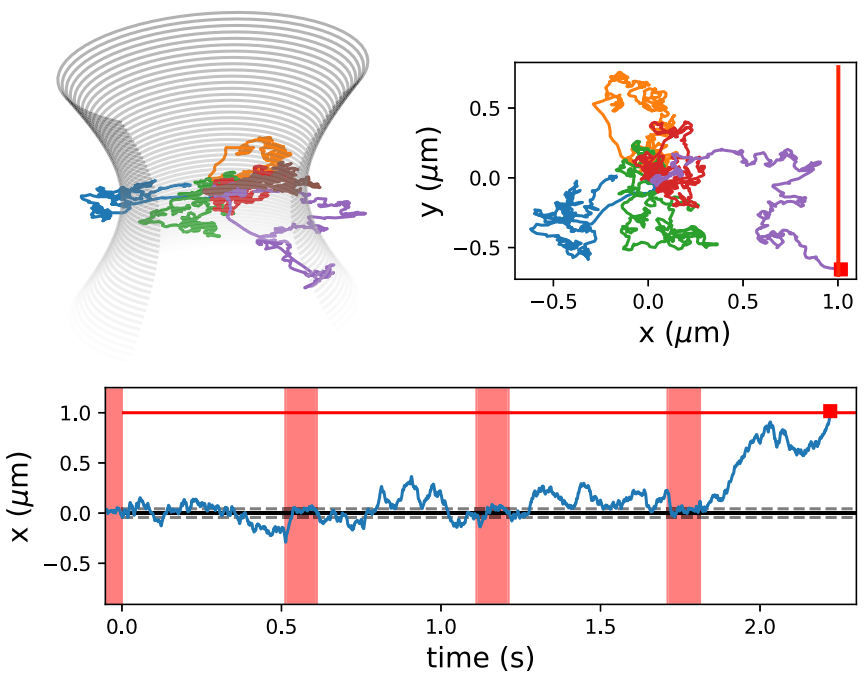

FIG. 1. Top panel: Sketch of the optical trap and a Brownian trajectory in the 2D plane $(x, y)$ with resetting. Bottom panel: Typical 1D Brownian trajectory with periodic resetting (red colored area) at time $T=0.5 \mathrm{~s}$. The equilibrium standard deviation of the trapped particle $\sigma=43 \mathrm{~nm}$ is shown (dotted lines). The target (red square $1 \mu \mathrm{m}$ away from the center of the trap) is reached when $x$ crosses the red line for the first time.

modulation (up to $10 \mathrm{kHz}$ ) or by means of an electro-optical modulator (EOM).

The position of the particle in the $(x, y)$ plane can be tracked (see Fig. 1) either by a white light imaging on a camera (maximum speed around 1000 frames per second) or from the deviation of a red laser on a quadrant photodiode (QPD) with a bandpass around $1 \mathrm{MHz}$. The accuracy of the measured position of the bead center is less than $1 \mathrm{~nm}$ for the QPD and about $2 \mathrm{~nm}$ for the camera. The bead was a few microns from the bottom surface of the cell to avoid errors in position reading due to sedimentation. Henceforth, for simplicity, we will focus only on the $x$ component of the particle's position, i.e., the one-dimensional (1D) trajectory. We can thus follow the particle position during its free diffusion and after a time $T$ reset its position by turning on the optical trap which allows us to realize different protocols.

Periodic resetting protocol. The experimental protocol leads us to study a model of diffusion in (effectively) one dimension subjected to periodic resetting. We consider an overdamped particle in thermal equilibrium inside a harmonic trap with potential $U(x)=\kappa x^{2} / 2$, where the stiffness $\kappa$ is proportional to the trapping laser power. This means that the initial position $x_{0}$ is distributed via the Gibbs-Bolzmann distribution which is simply Gaussian: $\mathcal{P}\left(x_{0}\right)=e^{-x_{0}^{2} / 2 \sigma^{2}} / \sqrt{2 \pi \sigma^{2}}$, with the width $\sigma=\sqrt{k_{B} \mathcal{T} / \kappa}$ where $\mathcal{T}$ is the temperature. We also consider a fixed target at location $L$. At time $t=0$, the trap is switched off for an interval $T$ and the particle undergoes free diffusion (overdamped) with diffusion constant $D=k_{B} \mathcal{T} / \Gamma$. At the end of the period, the particle's position is reset (see Fig. 1). Performing the resetting of the position poses the real experimental challenge.

In standard models of resetting one usually assumes instantaneous resetting [5], which is, however, impossible to achieve experimentally. There have been recent theoretical studies that incorporate a "refraction" period before the particle's position is reset [20,31-33]. In our experiment, we have an analog of this refraction period: After the period $T$, we switch on the optimal harmonic trap and we let the particle relax back to its equilibrium thermal distribution. This relaxation can, in principle, be made arbitrarily fast using, e.g., the recently developed "engineered swift equilibration" (ESE) technique [33-37]. In our experiment we determine the characteristic relaxation time inside the trap $\tau_{c}=\Gamma / k$. During the period $\left[T, T+\tau_{\text {eq }}\right]$ with $\tau_{\text {eq }} \simeq 3 \tau_{c}$, we do not make any measurement. In other words, even if the particle encounters the target during the relaxation period, we do not count that event as a first-passage event. Thus in this setup, the thermal relaxation mimics the instantaneous resetting, with one major difference, however. We do not reset it to exactly the same initial position-rather, the new "initial" position at the end of the time epoch $T+\tau_{\text {eq }}$ is drawn from the Gibbs-Boltzmann distribution $\mathcal{P}\left(x_{0}\right)$. At time $T+\tau_{\mathrm{eq}}$, we again switch off the trap and we let the particle diffuse freely for another period $T$, followed by the thermal relaxation over period $\tau_{\text {eq }}$. The process repeats periodically. During the free diffusion, if the particle finds the target at $L$, we measure the first-passage time $t_{f}$. Note that the first-passage time $t_{f}$ is the net "diffusion" time spent by the particle before reaching the target (not counting the intermediate relaxation periods $\tau_{\mathrm{eq}}$ ). Averaging over many realizations, we then compute the MFPT $\left\langle t_{f}\right\rangle$, for fixed target location $L$ and fixed resetting period $T$.

The MFPT for this protocol can be computed exactly, as detailed in the Supplemental Material [38]. Our main result can be summarized in terms of two dimensionless quantities,

$$
b=\frac{L}{\sigma} \quad \text { and } \quad c=\frac{L}{\sqrt{4 D T}} .
$$

The parameter $b$ quantifies how far the target is from the center of the trap in units of the trapped equilibrium standard deviation. The second parameter $c$ tells us how frequently we reset the particle compared to its free diffusion time. We show that the dimensionless MFPT $\tau$ can be expressed as a function of these two parameters $b$ and $c$,

$$
\tau=\frac{4 D\left\langle t_{f}\right\rangle}{L^{2}}=w(b, c),
$$

where

$$
w(b, c)=\frac{\int_{0}^{1} d v \int_{-\infty}^{\infty} d u e^{-u^{2} / 2} \operatorname{erf}\left(\frac{c}{\sqrt{v}}|1-u / b|\right)}{c^{2} \int_{-\infty}^{\infty} d u e^{-u^{2} / 2} \operatorname{erfc}(c|1-u / b|)} .
$$

While it is hard to evaluate the integrals explicitly, $w(b, c)$ can be easily plotted numerically to study its dependence on $b$ and $c$, as discussed later. Going beyond the first moment $\left\langle t_{f}\right\rangle$ and computing the full probability density function (PDF) of the first-passage time $t_{f}$ is also of great interest [39-42]. Indeed, we computed the PDF $F(t)$ of $t_{f}$ (see Supplemental Material [38]) and the result is plotted in Fig. 2. For small $b$, we found striking spikes in $F(t)$ just after each resetting event. We show in the Supplemental Material [38] that setting $t=$ $n T+\Delta$ with $n=0,1,2, \ldots$ and $\Delta \rightarrow 0^{+}$, the first-passage density $F(t=n T+\Delta)$ displays a power-law divergence (the 


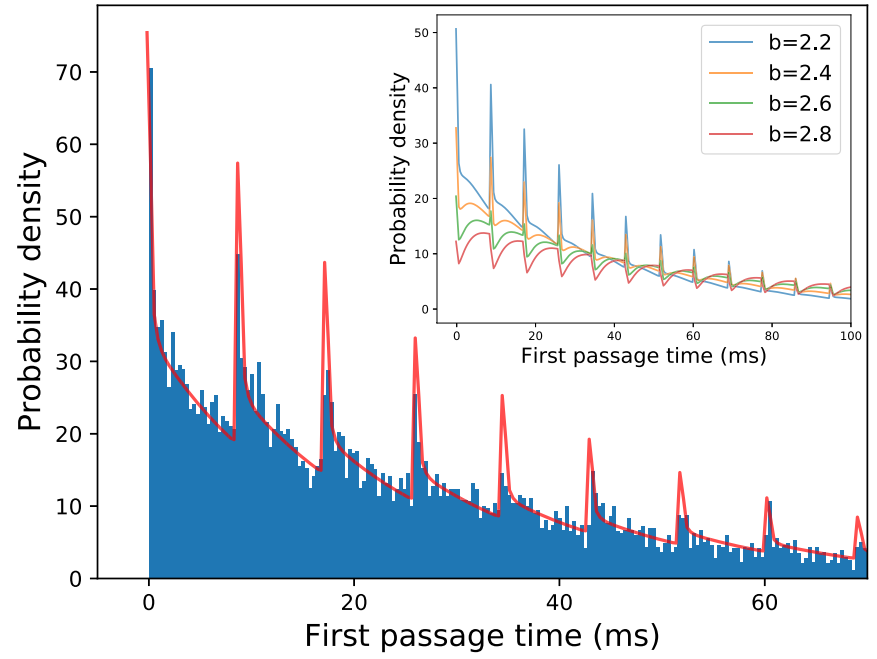

FIG. 2. Experimental (blue histogram) and theoretical (red line) PDF $F(t)$ of the first-passage time. We measured $1.2 \times 10^{4}$ firstpassage times for $b=2$ and $c=1(T=8.6 \mathrm{~ms})$. The spikes occur just immediately after $t=n T$ where $n=0,1,2, \ldots$ Inset: The PDF $F(t)$ vs $t$ (theoretical) for $b=2.2$ to 2.8 at $c=b / 2$ (i.e., $T$ constant) that demonstrates that the spikes disappear rapidly with increasing $b$. The PDF $F(t)$ is normalized such that $\int_{0}^{\infty} F(t) d t=1$ with $d t=$ $3.5 \times 10^{-4} \mathrm{~s}$.

spikes) as $\Delta \rightarrow 0^{+}$,

$$
F(t=n T+\Delta) \simeq A_{n}(b) \Delta^{-1 / 2},
$$

with an amplitude $A_{n}(b)$ that can be computed explicitly [38]. We find that as $b$ increases, $A_{n}(b)$ decays rapidly and the spikes disappear for large $b=L / \sigma$ (see the inset of Fig. 2). Instead, for large $b, F(t)$ drops by a finite amount after each period (as seen in the inset). In theoretical models of resetting to a fixed initial position $(\sigma=0$ or $b \rightarrow \infty)$, these spikes are completely absent and hence they are characteristic of the finiteness of $\sigma$.

In order to test experimentally these results we realized a periodic resetting protocol and measure the statistics of firstpassage times. The diffusion coefficient (typically $D \simeq 2 \times$ $10^{-13} \mathrm{~m}^{2} / \mathrm{s}$ ) is measured during the free diffusion part and the width of the Gaussian (typically $\sigma \simeq 40 \mathrm{~nm}$ ) when the particle is back at equilibrium. These independent and simultaneous measures allow us to overcome experimental drifts which may appear. In Fig. 2 we show the experimentally obtained PDF of $10^{4}$ measured first-passage times for $b=2$ and $c=1$. We also compared with our theoretical prediction [38]. We observe a very good agreement with no free parameter.

To analyze the MFPT, we start with the limit $b \gg 1$ of Eq. (3), i.e., $L \gg \sigma$. This limit corresponds to the case when the target is much farther away compared to the typical fluctuation of the initial position. In this case, taking the $b \rightarrow \infty$ limit in Eq. (3), we get

$$
\begin{aligned}
w(c) & =w(b \rightarrow \infty, c) \\
& =\frac{\operatorname{erf}(c)+2 c\left[e^{-c^{2}} / \sqrt{\pi}-c \operatorname{erfc}(c)\right]}{c^{2} \operatorname{erfc}(c)},
\end{aligned}
$$

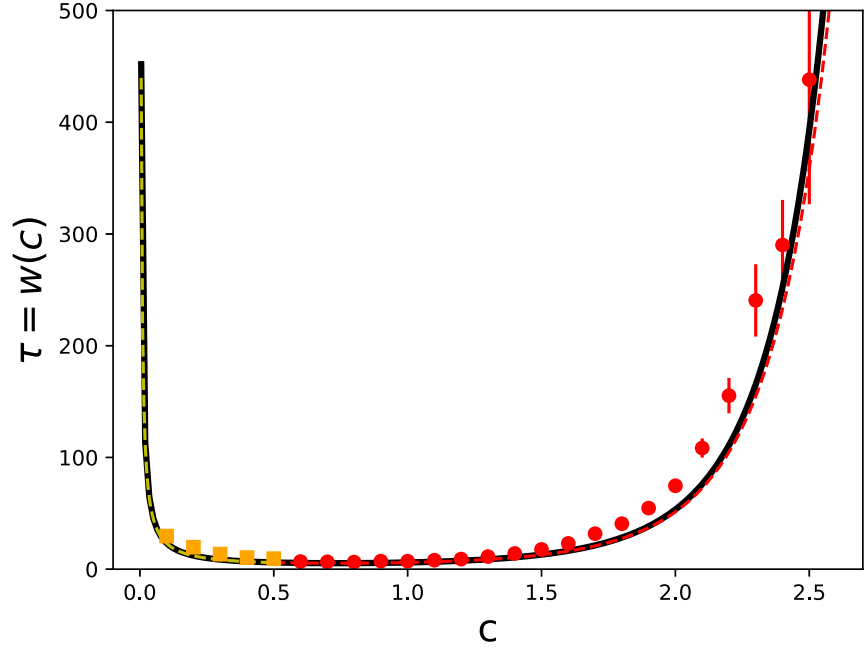

FIG. 3. $w(c) \equiv w(b \rightarrow \infty, c)$ vs $c$ curve (see also Fig. 4). The solid black line represents the theoretical formula for $w(c)$ in Eq. (5), while the dots denote the experimental data with $b=30$ (red dots) and $b=5$ (yellow square). The dotted lines show $w(b, c)$ with $b=$ 5 for small $c(c \leqslant 0.6)$ in yellow, and with $b=30$ for higher $c$ in red. The error bars are given by the standard deviation of the MFPT distribution divided by the square root of the number of events.

where $\operatorname{erf}(c)=(2 / \sqrt{\pi}) \int_{0}^{c} e^{-u^{2}} d u$ and $\operatorname{erfc}(c)=1-\operatorname{erf}(c)$. In Fig. 3, we plot $w(c)$ vs $c$ and compare with our experimental data and find good agreement with no adjustable parameter. Typically, to have a good estimate of the MFPT, we follow the particle for a few hours which allows us to detect between 1000 and 10000 first-passage times, depending on the values of $b$ and $c$. The standard deviation of first-passage times is of the same order as the MFPT. We see a distinct optimal value around $c^{*}=0.74$, at which $w\left(c^{*}\right)=5.3$. Our results thus provide a clear experimental verification of this optimal resetting paradigm. Let us remark that the authors in Ref. [9] studied periodic resetting to the fixed initial position $x_{0}=0$ and obtained the MFPT by a different method than ours. Our $b \rightarrow \infty$ limit result in Eq. (5) indeed coincides with that of Ref. [9], since when $\sigma \ll L$, our protocol mimics approximately a resetting to the origin.

What happens when $b=L / \sigma$ is finite? Remarkably, when we plot $w(b, c)$ in Eq. (3) as a function of $c$ for different fixed values of $b$, we see that $w(b, c)$ decreases as $c$ increases, indeed achieves a minimum at $c_{1}(b)$, then increases, achieves a maximum at $c_{2}(b)$, and then decreases monotonically as $c$ increases beyond $c_{2}(b)$ (see Fig. 4). When $b \rightarrow \infty$, the maximum at $c_{2}(b) \rightarrow \infty$ and one has a true minimum. However, for finite $b$, the "optimal" (minimal) MFPT at $c=c_{1}(b)$ is thus actually a metastable minimum and the true minimum occurs at $c \rightarrow \infty$, i.e., when the resetting period $T \rightarrow 0$. Physically, the limit $T \rightarrow 0$ corresponds to repeated (almost continuously) resetting and since the target position and the initial location are of the same order, the particle may find the target simply by resetting, without the need to diffuse. Interestingly, this metastable minimum exists only for $b>b_{c} \approx 2.3$. When $b<b_{c}$, the curve $w(b, c)$ decreases monotonically with $c$ and there is only a single minimum at $c \rightarrow \infty$, or equivalently for $T \rightarrow 0$. Thus the system undergoes a "first-order" phase 


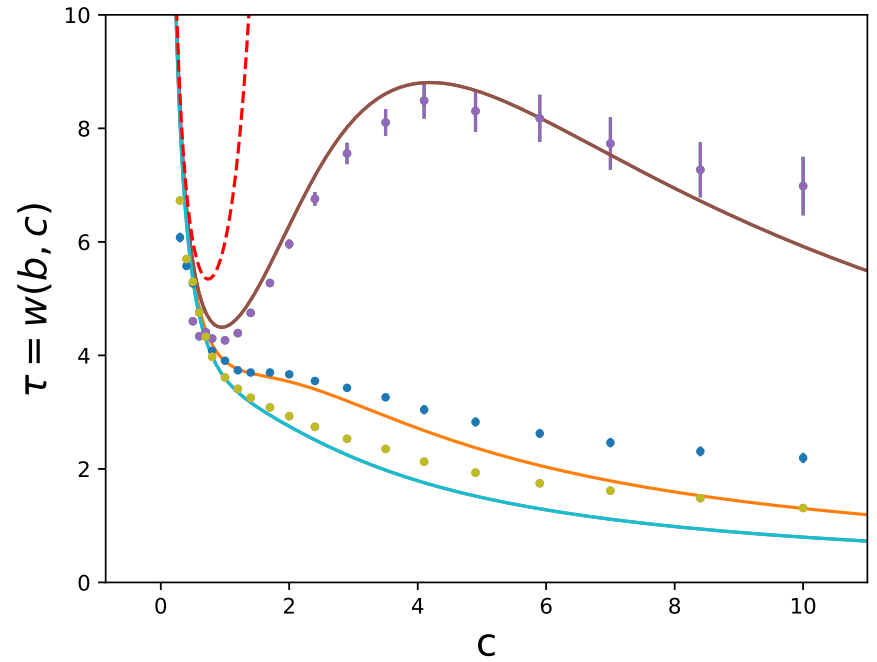

FIG. 4. The scaled MFPT $\tau=w(b, c)$ vs $c$ curves and experimental data for $b=3$ (brown curve and purple dots), $b=2.3$ (orange curve and blue dots), and $b=2$ (blue curve and yellow dots), for the periodic resetting protocol. The dotted curve recalls the $b \rightarrow \infty$ limit. The theoretical curves are obtained from Eq. (3). The scaled MFPT has two different behaviors depending on how far is the target. If $b>b_{c} \approx 2.3$, the MFPT $\tau$ exhibits a local minimum, whereas it decreases monotonically for $b$ lower than the critical value $b_{c}$.

transition as $b=L / \sigma$ is tuned across a critical value $b_{c} \approx 2.3$, from a phase with a metastable optimum at a finite $c=c_{1}(b)$ to one where the only minimum occurs at $c \rightarrow \infty$. This phase transition is well reproduced by the experimental data points. The deviation from theoretical predictions for high values of $c$ is due to the limited experimental acquisition rate (here, $50000 \mathrm{~Hz}$ ) that prevents us from detecting very fast events and thus leads to an overestimation of the MFPT as confirmed by our numerical simulations. This phase transition was rather unexpected and came as a surprise.

Random resetting protocol. It turns out that this metastability and the phase transition is rather robust and exists for other protocols, such as Poissonian resetting where the resetting occurs at a constant rate $r$. Here, the dimensionless variables are $b=L / \sigma$ and $c=\sqrt{r / D} L$ and the scaled MFPT $\tau=4 D\left\langle t_{f}\right\rangle / L^{2}$ again becomes a function $w_{2}(b, c)$ of $b$ and $c$ [analog of Eq. (3)]. In this case, we get a long but explicit $w_{2}(b, c)$ (see Supplemental Material [38] for details). In Fig. 5 we plot $w_{2}(b, c)$ vs $c$ for different values of $b$ together with the experimental data. We have a good agreement between theory and experiment and here again the deviation at high $c$ comes from a limited experimental acquisition rate. Once again, we see that there is a metastable minimum that disappears when $b$ decreases below a critical value $b_{c} \approx 2.53$. When $b \rightarrow$ $\infty$, there is only a single minimum at $c^{*}=1.59362$ where $w_{2}\left(\infty, c^{*}\right)=6.17655$. Thus this phenomenon of metastability and phase transition in MFPT seems to be robust.

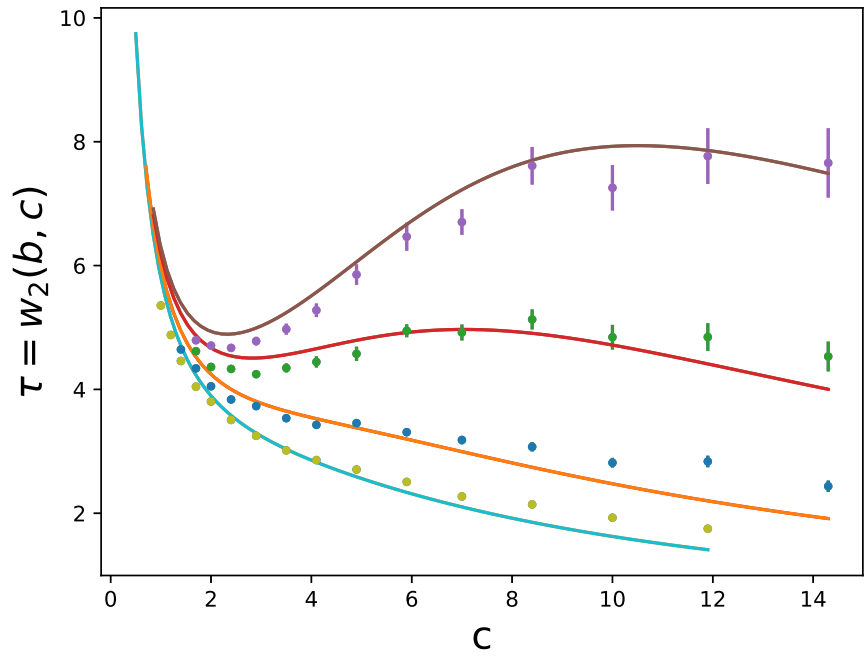

FIG. 5. Scaled MFPT $\tau=w_{2}(b, c)$ vs $c$ curves and experimental data points for $b=2,2.3,2.7$, and 3 (from bottom to top) in the case of Poissonian resetting. The metastable minimum disappears for $b<b_{c} \approx 2.53$.

Conclusions. We have studied both theoretically and experimentally the role of the variance of the resetting position on the optimal time needed for a Brownian bead to reach a specific immobile target. We have applied two different protocols, one with a periodic and another with a random resetting time. We found that both present a metastable minimum for $b>b_{c}$ (where $b_{c}$ depends on the protocol), showing that this transition could be a universal feature of MFPT protocols for resetting. For the periodic protocol, we also computed and measured the full PDF of the first-passage time and found that it displays striking spikes after each resetting event, a clear effect of the finiteness of the variance of the initial position. The experimental data agree well with theoretical predictions, but the experiment is not a mere test of the theory. Indeed, we point out a series of experimental difficulties that one has to consider when one applies such theoretical predictions in real systems. On one side, when the particle is free for a very long time (small $c$ ) there are problems of sedimentation that must be taken into account because they may influence greatly the final result. On the very short times (large $c$ ) we have shown that finite sampling time affects the results, because if the first passage is not detected, it leads to an overestimation of the first-passage time. It is clear that at very fast sampling rates the model will fail and probably the role of inertia, which has been always neglected, should be taken into account in future theoretical developments.

Acknowledgments. We thank E. Trizac for useful discussions. S.N.M. wants to thank the warm hospitality of International Centre for Theoretical Sciences (Bangalore) where this work was completed.
[1] M. Villen-Altramirano and J. Villen-Altramirano, RESTART: A method for accelerating rare event simulations, in Queueing,
Performance and Control in ATM, edited J. W. Cohen and C. D. Pack (North-Holland, Amsterdam, 1991). 
[2] M. Luby, A. Sinclair, and D. Zuckerman, Inf. Proc. Lett. 47, 173 (1993).

[3] H. Tong, C. Faloutsos, and J.-Y. Pan, Knowl. Inf. Syst. 14, 327 (2008).

[4] J. H. Lorenz, Runtime distributions and criteria for restarts, in SOFSEM 2018: Theory and Practice of Computer Science, edited by A Tjoa, L. Bellatreche, S. Biffl, J. van Leeuwen, and J. Wiedermann, Lecture Notes in Computer Science Vol. 10706 (Springer, Berlin, 2018).

[5] M. R. Evans, S. N. Majumdar, and G. Schehr, J. Phys. A: Math. Theor. 53, 193001 (2020).

[6] O. Bénichou, C. Loverdo, M. Moreau, and R. Voituriez, Rev. Mod. Phys. 83, 81 (2011).

[7] M. R. Evans and S. N. Majumdar, Phys. Rev. Lett. 106, 160601 (2011).

[8] M. R. Evans and S. N. Majumdar, J. Phys. A: Math. Theor. 44, 435001 (2011).

[9] A. Pal, A. Kundu, and M. R. Evans, J. Phys. A: Math. Theor. 49, 225001 (2016).

[10] U. Bhat, C. De Bacco, and S. Redner, J. Stat. Mech. (2016) 083401.

[11] M. R. Evans, S. N. Majumdar, and K. Mallick, J. Phys. A: Math. Theor. 46, 185001 (2013).

[12] M. Montero and J. Villarroel, Phys. Rev. E 87, 012116 (2013).

[13] M. R. Evans and S. N. Majumdar, J. Phys. A: Math. Theor. 47, 285001 (2014).

[14] L. Kuśmierz, S. N. Majumdar, S. Sabhapandit, and G. Schehr, Phys. Rev. Lett. 113, 220602 (2014).

[15] S. Reuveni, M. Urbakh, and J. Klafter, Proc. Natl. Acad. Sci. USA 111, 4391 (2014).

[16] T. Rotbart, S. Reuveni, and M. Urbakh, Phys. Rev. E 92, 060101(R) (2015).

[17] C. Christou and A. Schadschneider, J. Phys. A: Math. Theor. 48, 285003 (2015).

[18] L. Kuśmierz and E. Gudowska-Nowak, Phys. Rev. E 92, 052127 (2015).

[19] A. Nagar and S. Gupta, Phys. Rev. E 93, 060102(R) (2016).

[20] S. Reuveni, Phys. Rev. Lett. 116, 170601 (2016).

[21] A. Pal and S. Reuveni, Phys. Rev. Lett. 118, 030603 (2017).
[22] A. Chechkin and I. M. Sokolov, Phys. Rev. Lett. 121, 050601 (2018).

[23] S. Belan, Phys. Rev. Lett. 120, 080601 (2018).

[24] M. R. Evans and S. N. Majumdar, J. Phys. A: Math. Theor. 51, 475003 (2018).

[25] A. Masó-Puigdellosas, D. Campos, and V. Méndez, Phys. Rev. E 99, 012141 (2019).

[26] J. Masoliver and M. Montero, Phys. Rev. E 100, 042103 (2019).

[27] X. Durang, S. Lee, L. Lizana, and J.-H. Jeon, J. Phys. A: Math. Theor. 52, 224001 (2019).

[28] A. Pal and V. V. Prasad, Phys. Rev. E 99, 032123 (2019).

[29] O. Tal-Friedman, A. Pal, A. Sekhon, S. Reuveni, and Y. Roichman, arXiv:2003.03096.

[30] A. Bèrut, A. Imparato, A. Petrosyan, and S. Ciliberto, J. Stat. Mech. (2016) 054002.

[31] M. R. Evans and S. N. Majumdar, J. Phys. A: Math. Theor. 52, 01LT01 (2019).

[32] A. Pal, L. Kusmierz, and S. Reuveni, Phys. Rev. E 100, 040101(R) (2019).

[33] D. Gupta, C. A. Plata, and A. Pal, Phys. Rev. Lett. 124, 110608 (2020).

[34] I. A. Martinez, A. Petrosyan, D. Guery-Odelin, E. Trizac, and S. Ciliberto, Nat. Phys. 12, 843 (2016).

[35] D. Guery-Odelin, A. Ruschhaupt, A. Kiely, E. Torrontegui, S. Martinez-Garaot, and J. G. Muga, Rev. Mod. Phys. 91, 045001 (2019).

[36] C. A. Plata, D. Guery-Odelin, E. Trizac, and A. Prados, Phys. Rev. E 99, 012140 (2019).

[37] M. Chupeau, B. Besga, D. Guery-Odelin, E. Trizac, A. Petrosyan, and S. Ciliberto, Phys. Rev. E 98, 010104(R) (2018).

[38] See Supplemental Material at http://link.aps.org/supplemental/ 10.1103/PhysRevResearch.2.032029 for details on how the theoretical predictions plotted in Figs. 2-4 have been obtained, both for periodic and random resetting.

[39] S. N. Majumdar, Curr. Sci. 77, 370 (1999).

[40] S. Redner, A Guide to First-Passage Processes (Cambridge University Press, Cambridge, UK, 2001).

[41] S. N. Majumdar, Physica A 389, 4299 (2010).

[42] A. J. Bray, S. N. Majumdar, and G. Schehr, Adv. Phys. 62, 225 (2013). 